Qualitative Health Research, 1049732312470761, first published on December 28, 2012 as doi:10.1177/1049732312470761

\title{
Malaria "Diagnosis" and Diagnostics in Afghanistan
}

Joanna Reynolds, ${ }^{1}$ Molly Wood, ${ }^{2}$ Amy Mikhail, ${ }^{2}$ Tamanna Ahmad, $^{2}$ Karimullah Karimullah, ${ }^{2}$ Mohibullah Motahed, ${ }^{2}$ Anwar Hazansai, ${ }^{3}$ Sayed Habib Baktash, ${ }^{4}$ Nadia Anwari, ${ }^{2}$ James Kizito, ${ }^{5}$ Ismail Mayan, ${ }^{2}$ Mark Rowland ${ }_{1}^{1}$ Clare Chandler, ${ }^{1}$ and Toby Leslie ${ }^{1}$

${ }^{1}$ London School of Hygiene \& Tropical Medicine, London, United Kingdom

${ }^{2}$ Health Protection and Research Organisation, Kabul, Afghanistan

${ }^{3}$ HealthNet TPO, Kabul, Afghanistan

${ }^{4}$ MERLIN, Kabul, Afghanistan

${ }^{5}$ Infectious Diseases Research Collaboration, Kampala, Uganda

\section{Corresponding Author:}

Joanna Reynolds, London School of Hygiene \& Tropical Medicine, Department of Global Health \& Development, 15-17 Tavistock Place, London WC1H 9SH, UK

Email: joanna.reynolds@lshtm.ac.uk 


\section{Abstract}

In many malaria-endemic areas, including Afghanistan, overdiagnosis of malaria is common. Even when using parasite-based diagnostic tests prior to treatment, clinicians commonly prescribe antimalarial treatment following negative test results. This practice neglects alternative causes of fever, uses drugs unnecessarily, and might contribute to antimalarial drug resistance. We undertook a qualitative study among health workers using different malaria diagnostic methods in Afghanistan to explore perceptions of malaria diagnosis. Health workers valued diagnostic tests for their ability to confirm clinical suspicions of malaria via a positive result, but a negative result was commonly interpreted as an absence of diagnosis, legitimizing clinical diagnosis of malaria and prescription of antimalarial drugs. Prescribing decisions reflected uncertainty around tests and diagnosis, and were influenced by social- and health-system factors. Study findings emphasize the need for nuanced and context-specific guidance to change prescriber behavior and improve treatment of malarial and nonmalarial febrile illnesses.

\section{Keywords}

Asia, Central; developing countries; health care professionals; illness and disease, infectious; interpretive methods; interviews; technology, medical 
Malaria causes an estimated 800,000 deaths and 225 million clinical cases per year (World Health Organization, 2010a). Although most of these occur in Africa, around two billion people are thought to be at risk of malaria infection in South and West Asia, including large areas of India, Pakistan, and Afghanistan (Guerra et al., 2010). In these areas, overdiagnosis and misdiagnosis of malaria is common (Whitty, Chandler, Ansah, Leslie, \& Staedke, 2008). In many places, prevalence of malaria as a proportion of febrile illness is falling, and the introduction of more costly artemisinin-based combination therapies (ACTs) for malaria has increased the emphasis on appropriate parasite-based diagnosis of malaria for accurate treatment. Accurate diagnosis and treatment of malaria in nonspecific fever cases is important in delaying drug resistance, preventing wastage of resources, improving case management of nonmalarial fevers, and for disease surveillance (Kyabayinze et al., 2010; Perkins \& Bell, 2008). The use of parasite-based malaria diagnosis via either microscopy or malaria rapid diagnostic tests (RDTs) prior to prescription of antimalarial drugs is now a central component of global guidelines for malaria treatment (World Health Organization, 2010b).

The introduction of parasite-based diagnosis on its own does not guarantee accurate treatment, however. Prescribers often treat patients with antimalarial drugs despite the presence of a negative parasitological diagnosis (Ansah et al., 2010; Reyburn et al., 2007). Thus, alternative causes of fever are overlooked and the potential cost effectiveness of the diagnostic technologies is undermined (Lubell et al., 2008). Influencing prescribers' behaviors and improving adherence to test results is therefore an important component of improving treatment of febrile illness in low-resource settings (Bisoffi, Gobbi, Angheben, \& Van den Ende, 2009; Chandler, Whitty, \& Ansah, 2010; Kyabayinze et al., 2010).

Malaria RDTs are being widely introduced to meet the requirements of global treatment guidelines. They are considered easy, quick, and accurate, and particularly useful in low-resource settings where microscopy might be unavailable or unwarranted (Kyabayinze et al., 2010). With training most often limited to the mechanics of performing the tests, the tests alone do not appear to improve health workers' prescribing behaviors when compared to microscopy in most settings (Bisoffi et al., 2009; Chandler et al., 2010; Kyabayinze et al.). Broader social and material relations appear to be critical factors in improving diagnostic and treatment behaviors. These might include conceptualizations of diagnostic technologies, medicines, disease, and treatment; interactions between health workers and patients, and within communities of health workers; and how these are situated within the wider health system (Chandler et al., 2010; Cohen, Dupas, \& Schaner, 2011).

To date, the issues surrounding health workers' use of malaria diagnostic tools and subsequent treatment practices have been explored in some depth in various settings in sub-Saharan Africa (Ansah et al., 
2010; Chandler et al., 2008; Chandler et al., 2010; Cohen et al., 2011; Harvey et al., 2008; Kyabayinze et al., 2010). However, there is limited similar literature applicable to malaria-endemic countries outside this region, including Afghanistan. Hence, in this article we describe a qualitative study conducted to explore health workers’ conceptualizations of RDTs in relation to microscopy and clinical diagnosis of malaria in Afghanistan. The aim was to understand what influences health workers' diagnostic and prescribing behaviors in relation to malaria in the public primary health care system.

The theoretical orientation for this study was based on our interpretive perspective, seeking to explore the meaning of malaria diagnosis and treatment in practice and in relation to the broader social and political context. In the theoretical framing of this study we drew on anthropological literature, the assumption that there are multiple realities of a single disease or medical condition, and that these are enacted through the care process (Mol, 2002). The objects of medicine, including diagnostic technologies, are considered important in the study of the patient care process. Focus on these has enabled exploration of the relationship between health worker and patient, and how knowledge of the body and disease is constituted within the care context (Lock \& Nguyen, 2010). As Gardner, Dew, Stubbe, Dowell, and Macdonald (2011) argued, it is through practicalities, such as the practical use of technologies and the establishment of material relations, that aspects of the body and disease become knowable and diagnosis can take place.

Exploring health workers' experiences of engaging with diagnostic tools illuminated understandings of the process by which malaria diagnoses were constructed and interpreted by health workers within the context of the competing priorities encountered in their daily practice. We were also informed by literature examining the symbolic and communicative properties of medicines and prescriptions, which can alleviate clinical uncertainty and bring resolution to the clinical encounter (Reynolds Whyte, Van der Geest, \& Hardon, 2002). As such, we aimed to explore the roles of diagnostics and medicine in the construction and negotiation of the patient care process (Nichter \& Vuckovic, 1994). We sought to locate Afghan health workers’ conceptualizations of malaria diagnosis and treatment within this body of theory.

\section{Methods}

\section{The Health Care Context}

In Afghanistan, malaria is endemic to large areas at altitudes below 2,000 meters, resulting in nearly $80 \%$ of the population being at risk of transmission (Brooker et al., 2006; World Health Organization, 2010c). Malaria is considered a public health concern and its control is one of seven priority areas covered by the public health system (Ministry of Public Health, 2007). Primary health care is delivered via the Basic Package of Health 
Services (BPHS) and implemented by contracted nongovernmental organizations (Morikawa, Schneider, Becker, \& Lipovac, 2011; Trani, Bakhshi, Noor, Lopez, \& Mashkoor, 2010). There are four main levels of health facility, the first three offering primary care, and the last, referral care: health posts, basic health centers (BHCs), comprehensive health centers (CHCs), and district hospitals.

Health personnel working at BHCs and CHCs were included in this study. A BHC is usually staffed by one doctor and a nurse or midwife, whereas a CHC has two or three doctors and several nursing staff. All CHCs have laboratories that provide, among other services, malaria diagnosis through microscopy. Microscopy is not generally available in all BHCs because it is not mandated by the BPHS, but it is applied by some implementers where malaria is endemic, including in our study clinics. All clinics in our study also had access to RDTs as part of the broader trial (see below).

Malaria incidence is variable across Afghanistan. Rates have been highly changeable over the past few decades as a result of conflict, fragmentation of public health infrastructure, movement of populations, and changes in land use (e.g., intensified rice cultivation; Adimi, Soebiyanto, Safi, \& Kiang, 2010; Howard, Shafi, Jones, \& Rowland, 2010). Recent malaria control activities have included indoor residual spraying programs and distribution of insecticide-treated nets (Howard et al., 2010; Ministry of Public Health, 2007). However, these efforts are challenged by lack of infrastructure and continuing extreme poverty (Afghanistan Independent Human Rights Commission, 2011; Howard et al., 2003).

Unlike in sub-Saharan Africa, Plasmodium vivax is the dominant form of malaria infection in Afghanistan. Although rates of the more dangerous Plasmodium falciparum have varied considerably, only around 6\% of nationally reported malaria cases in 2009 resulted from this species (Adimi et al., 2010; World Health Organization, 2010c). Appropriate diagnosis and treatment of malaria is crucial in this setting because treatment differs for the two species. ACTs should be prescribed for falciparum cases only, and the cheaper chloroquine for vivax malaria (Leslie et al., 2007).

The seasonality and low prevalence of malaria means that only a minority of febrile illnesses are the result of malaria infections (Rowland et al., 2002). In spite of this, suspected malaria is most often diagnosed presumptively in Afghanistan (Ministry of Public Health, 2007). In low-prevalence areas, where the proportion of patients with fever who have malaria is less than $1 \%$, the reliance on clinical diagnosis results in more than 95\% of patients who do not have malaria receiving antimalarial drugs. Parasite-based diagnostic tests have improved this significantly, but $20 \%$ to $30 \%$ of patients testing negative under microscopy are still treated with antimalarial drugs (Leslie et al., 2012). 


\section{Study Sites}

We carried out a qualitative study alongside a trial to introduce RDTs in two provinces in Afghanistan: one in the north, and a second in the east. Both provinces are considered high priority for malaria risk, although disease transmission differs significantly between sites (Ministry of Public Health, 2007). The results of an observational study conducted between July and September 2009 indicated that in the northern province, fewer than $1 \%$ of patients tested for malaria were positive under microscopy, compared with $10 \%$ to $25 \%$ in the eastern province (Leslie et al, 2012). We conducted this study at a time when health care in Afghanistan was undergoing rapid expansion in coverage. In the study areas, the number and range of health facilities has increased in recent years: in the northern province, the number of health clinics increased from 20 in 2005 to 52 in 2011; in the eastern province, district hospitals doubled from two in 2005 to four in 2011.

A trial to assess the effect of the introduction of RDTs on prescriber practices was conducted at health facilities in the northern and eastern provinces in 2010-2011. In this trial, patients with suspected malaria were individually randomized to different diagnostic methods. The intervention method was to test for malaria by RDT; the control method was the usual diagnosis method for the facility, which varied between provinces and health facilities. In the eastern province, microscopy was considered usual practice, having been in place in all participating health facilities for more than 5 years (labeled "established microscopy"). In the northern province, for half of the participating facilities, microscopy was relatively new (“new microscopy”), available since 2009, whereas the remaining facilities had no access to microscopy, relying only on clinical judgment (“clinical judgment”). The aim of the trial was to assess the accuracy of treatment prescribed under the three diagnostic scenarios: RDTs, microscopy, and clinical judgment.

\section{The Qualitative Study}

We designed a qualitative study to explore conceptualizations of the processes of malaria diagnosis and treatment among health workers who had been part of the trial, and their experiences of new diagnostic tests. Patients' expectations, perceptions, and experiences of consultations, when presenting with fever, were also explored through entry and exit interviews, and will be reported separately (Chandler et al., in preparation). We used the trial database to identify and invite all health workers who had prescribed to more than 10 patients during the trial, including medical doctors, nurses, and midwives, to participate in in-depth interviews. Participants were given information about the study and provided their consent for voluntary participation. The interview topic guide consisted of a series of semistructured and open-ended questions and was designed to elicit narratives through which participants could reflect on and make sense of their experiences of using (or not 
using) diagnostic tools in their practice. The topic guide was updated iteratively throughout the data collection process to reflect queries emerging from the ongoing analysis.

Ethical approval for the study was given by the Institutional Review Board of the Ministry of Public Health, Afghanistan and the London School of Hygiene \& Tropical Medicine Ethics Committee. A team of native Pashto- and Dari-speaking local field workers were given intensive training in methods and objectives of the study. One field worker of each gender carried out the interviews at each site, between October 2010 and March 2011. The interviews were recorded (with participants' permission), transcribed verbatim, and translated into English using a meaning-based approach by bilingual members of the local research team. The field coordinator checked all translations for accuracy and revised them in collaboration with the local team. Demographic information about each respondent was entered into an Excel spreadsheet.

We started data analysis alongside data collection. We used a bottom-up approach to code ideas in the transcripts line by line, grouping these ideas together into themes and theoretical constructs in an ongoing process during the coding, informed by our theoretical orientation (Auerbach \& Silverstein, 2003). This method reflected a more theoretical approach than thematic or content analysis, and was informed by the iterative approach of grounded theory analysis (Strauss \& Corbin, 1998). The development of a coding template was led by Joanna Reynolds, who worked with two social science research assistants to code the transcripts and continuously update the template to reflect emerging themes and constructs. Agreement on codes and the template was reached through frequent discussions of the meanings of codes, themes, and constructs, and differences between coder interpretations. Following coding of all the transcripts, we explored themes and constructs across the data, and discussed interpretation of these with the broader study team to ensure appropriateness of interpretation.

Results

The field team conducted in-depth interviews with 39 health workers in 22 health facilities, 10 in the northern province and 12 in the eastern province. The clinics were a mixture of BHCs $(n=15)$ and CHCs $(n=7)$, and participants included doctors, nurses, medical assistants, one assistant doctor (or feldsher, an assistant physician trained under the Russian system) and one community health supervisor. The mean number of years of professional experience was very similar in both provinces (approximately 12 years), and this reflected the typical age of clinicians working in these types of health centers, who had often completed several years of practice in higher-level facilities before taking up these positions (see Table 1 for a summary of the participants). Based on feedback and discussion with the field team conducting the interviews, minor 
adjustments were made to the topic guide throughout the data collection, but particularly following the first 11 interviews to explore more fully themes that were emerging from the ongoing data analysis.

INSERT TABLE 1 ABOUT HERE.

As an overview, the results presented two distinct but concurrently held conceptualizations of the role of parasite-based testing in relation to malaria diagnosis and treatment. Positive test results from microscopy and RDTs were perceived as valuable for enabling a confirmed diagnosis of malaria. This was interpreted as both reflecting and reinforcing health workers' clinical diagnostic skills, instilling confidence in subsequent treatment. Health workers also conceptualized an important role for tests beyond clinical outcomes, in managing limited resources and satisfying patients' expectations of care.

At the same time, common conceptualizations of a negative test result were that it represented an "absence of diagnosis", the recognition of which appeared to legitimize the (re)consideration of symptoms and use of a clinical diagnosis of malaria. The concept of the "absence of diagnosis" also highlighted the range of broader contextual factors that influenced the process of diagnosis and the subsequent decision to treat. Although there were some differences between perceptions of microscopy and RDTs, as detailed below, many participants appeared to conflate the two technologies when discussing tests and testing. In addition, there appeared to be few noticeable differences across the data between responses from the two provinces or the three trial arms, suggesting minimal impact of the different diagnostic methods on perceptions of testing in general.

\section{The Value of Testing Lies in the Confirmed Diagnosis}

Participants perceived a range of benefits of using microscopy and RDTs over diagnosis through clinical judgment of symptoms. The benefit of a test result centered on the construct of a confirmed diagnosis of malaria, whereby a positive test result was perceived to evoke confidence in the clinically based diagnosis of malaria, and guided the subsequent prescription of antimalarial drugs. This was conveyed by a community health supervisor from the northern province:

Interviewer (I): Did you bring any significant changes to your diagnostic methods during last year in comparison with when you didn't have the RDT?

Participant (P): Yes, because we didn’t have a laboratory here before. When the patients said their signs and symptoms to me I gave medicine based on clinical judgment. Now here there is microscopy and other things. I do confirmed diagnosis then I prescribe medicine for them. There is a change here in diagnosis. 
Through this construct of confirmed diagnosis participants conveyed two ideas: validation of their own clinical ability to detect malaria, and confidence in the effectiveness or appropriateness of treatment subsequently prescribed.

Tests can confirm my clinical diagnosis. The construct of confirmed diagnosis of malaria appeared to comprise the concurrent use of both clinical judgment and a confirmatory test result. Notions of certainty appeared embedded in the idea of the confirmed diagnosis, suggesting that tests were valued for their ability to evoke confidence in the health workers' initial diagnosis. A doctor from the northern province said,

When there was no RDT here we prescribed antimalarials for patients with doubt, and we were not certain one hundred percent about the patients if they had malaria or not, although [the northern province] is an endemic malarial region. And when the RDT was brought to our clinic I became certain that the patient has malaria or not. It was confirmed by RDT.

The role of testing, then, seemed to confirm what was already clinically suspected or known by the health worker. The majority of participants described the test in terms of its value in verifying their clinical judgment of malaria. As a nurse from the northern province stated, health workers "diagnose[d] clinically according to the symptoms and signs and confirm[ed] by RDT or microscopy.” Hence, it was not the diagnostic test in itself that produced a malaria diagnosis, but the process of the test confirming the clinical skills of the health worker, and their judgment of malaria.

Tests can provide certainty for treatment. The confirmed diagnosis was considered of central importance in providing treatment for malaria-positive patients. Among some participants the confirmed diagnosis was also valued for the test's ability to detect the type of malaria, enabling them to prescribe the most appropriate treatment for the patient. The confidence with which treatment could be prescribed following a diagnostic test was related to the notion of avoiding harm associated with prescribing unnecessary medicines. For example, a few health workers described antimalarials and other drugs as harmful, even poisonous, as well as having curative properties. A doctor from the eastern province said,

In general, the man who does not have malaria but expects himself to have malaria, he should be first made aware about the meaning of drug poison; that drugs also give poison to the human. So the good thing is to prove the patient's malaria first, and then medicines should be given. 
Hence, the role of testing in facilitating the correct prescription of medicine for a patient reflected both the ideas of enabling the patient to recover from the disease and avoiding the harm inherent in providing unnecessary drugs.

Testing satisfies patients. A number of participants perceived laboratory testing in general to be considered by patients to be an important part of the consultation, and something patients often expected and requested. Health workers suggested that testing for malaria appeared to be a significant factor in patients' satisfaction with the care they received, and might have influenced their perceptions of the effectiveness of treatment prescribed. A nurse from the northern province stated,

[Using tests] has good effect on treatment as well as on patients, because the doctor is confident in his diagnosis on the basis of lab [laboratory] results and the patient is also happy with the lab results and knows that the medicine will be effective, on the basis of accurate diagnosis. Here is the rural area and people give much value to the lab tests.

Hence, testing played a role in the social as well as clinical interaction with patients, and was related by health workers to perceived patient satisfaction.

Tests can help in resource management. Beyond the immediate clinical benefits of using tests, health workers identified their potential role in addressing health systems issues that were seen to affect the delivery of care. Several health workers acknowledged the ability of tests, whether microscopy or RDTs, to reduce the number of malaria treatments prescribed, thus playing a role in the management of limited supplies of drugs. These health workers articulated a responsibility to not overprescribe medication. They highlighted the importance of adhering to a test result to avoid "the unreasonable use of medicines," as stated by a doctor from the eastern province.

RDTs in particular were associated with improving access to good care. First, the perceived ease of using RDTs was cited by a number of participants as a way to increase patients' access to good diagnoses in remote areas served only by community health workers, and/or where there was no microscopy. Second, health workers perceived RDTs to be beneficial in helping reduce the burden on laboratories by decreasing the demand for microscopy. This was presented against a described context of poorly resourced laboratories that were not always able to cope with the levels of testing required, as conveyed by a doctor from the eastern province: I mean, if the rapid diagnostic tests are widely introduced then people can test their blood and bring their RDT strip and directly tell me that I have malaria and now give me medicines for treatment. 
Because the people are poor and the health facilities in Afghanistan are free, they will come to the clinic for medicines and they will be less crowded here, and also the lab technician will have time to do other tasks.

Here, the participant related the value of RDTs to the wider context of health care provision and acknowledged the position of malaria care alongside other demands on the time and resources of laboratories.

RDTs improve efficiency. RDTs were valued for their physical qualities, which were considered to make a confirmed diagnosis easier and quicker to achieve than when using microscopy. The RDT was praised by some for its completeness in providing all necessary items the health worker needed to conduct the test. The RDT was also valued above microscopy for its speed; the RDT produced a test result much more quickly than a slide and the health worker did not have to rely on the skills of another person or the availability of laboratory equipment. A doctor from the eastern province said,

You can see that the [rapid diagnostic] tests will prevent slide drying, staining, artifacts, and reading errors. ... The diagnosis is made exactly and also has no error. Maybe the lab technician has no experience and may make the wrong diagnosis, but by these means, the same [correct] diagnosis is made.

Having RDTs available at the facility was considered an important aspect of the process by which to treat malaria, because "the patient can get medicines in much less time,” according to another doctor from the eastern province, thus increasing the efficiency of the care process.

\section{A Negative Result is Not a Diagnosis}

Participants' descriptions of the value of testing for malaria tended to be abstract and theoretical, emerging in response to general questions about perceptions of diagnostic technologies. However, many health workers appeared to challenge and undermine these statements when asked about their own practical experiences in responding to test results. Subsequently, clinical judgment of malaria emerged as a justifiable response to a negative test result. This negative result was perceived as representing the absence of a diagnosis at that stage in the clinical encounter.

Absence of diagnosis can lead to treatment for clinical malaria. When the test result was negative the need to determine a diagnosis remained. Health workers listed other possible diagnoses they would consider when faced with a negative malaria test result, which covered a wide range of conditions, including respiratory 
conditions, typhoid, dysentery, and urinary tract infections, among others. Many participants were vague in describing how they would come to an alternative diagnosis. For example, a medical assistant from the northern province explained little beyond "see[ing] the signs and symptoms," and many others stated that there were few means available to them to test for other causes, including a doctor from the eastern province: "We cannot make another kind of diagnosis for the patient because our laboratory function is very limited, and we cannot diagnose the other diseases because it needs a well-facilitated laboratory to diagnose other diseases.”

Several treatment options were identified by health workers as commonly used for these diseases, including antibiotics and analgesics. However, most participants also described having prescribed antimalarial drugs following a negative malaria test result. A common framing of a negative test result was that of the “absence of a diagnosis," rather than confirmation that the patient did not have malaria. As such, in many participants' accounts, a negative test did not rule out the possibility of a malaria diagnosis, as indicated by a doctor from the eastern province:

I: Has this happened, when you suspected the RDT result?

P: Why not? It has happened many times, when the RDTs have given no result. . . Then we have included such a patient in clinical malaria and have written for him [prescribed] medicines.

Salient across many participants' accounts of their practice was the notion that a negative result triggered a reconsideration of clinical malaria alongside a range of other possible diagnoses. Following this, typically, was a reemphasis on the importance of clinical judgment. Most health workers described experiences of reexamining the patient's symptoms following a negative test, diagnosing clinical malaria and then prescribing antimalarial drugs. This was exemplified by a nurse from the eastern province:

So mostly they have negative result on microscopy. ... The result comes out negative, but when we see the signs and symptoms and these all are exactly that of malaria, and [the patient] has no signs and symptoms of any other disease, so then we are obligated to make diagnosis for malaria because the patient tells us all the right symptoms of malaria.

The word "obligated" in the above quotation indicated that the health worker had no option but to make a diagnosis, and in the absence of a confirmed diagnosis of malaria or symptoms indicating another disease, a clinical diagnosis of malaria was valid. Prescription of antimalarial drugs following this seemed, therefore, a 
logical step in the process of clinical care. It was portrayed as such in many participants' accounts of responding to a negative test result, including a doctor from the eastern province:

I: Okay doctor, in your practice, when a patient's lab test for malaria is negative, then what do you do with such patients?

P: On the basis of signs and symptoms, we make the clinical judgment, suspected malaria, and then we start the medication.

Thus it appeared that, in practice, clinical malaria coexisted with parasitologically confirmed malaria within the context of caring for febrile patients, and provided the logic for the prescription of antimalarial drugs following a negative test result. Both parasite-based and clinical diagnoses were engaged in the decision to treat a patient with a negative test result.

Doubts about tests. The concept of the absence of a diagnosis following a negative result emerged in addition to doubts about the accuracy of tests, based on hypotheses about parasites, patients, and laboratory staff, and the intrinsic quality of the test itself. A commonly identified issue for microscopy, and also sometimes for RDTs, was the possibility of the parasites, or signs of malaria, being undetectable in the blood sample taken. This was hypothesized to occur sometimes as a result of patient actions. For example, a doctor from the eastern province indicated that a patient taking an antimalarial before attending the facility might have "hidden the signs of ... malaria," affecting the ability of either microscopy or RDTs to detect malaria.

Wider contextual constraints on the resources needed for providing accurate diagnoses were also implicated in doubts cast over the reliability of negative test results. A lack of good-quality equipment or electricity affected trust in microscopy results, and some health workers doubted microscopy because of its reliance on human ability. Participants, such as a doctor from the northern province, explained that sometimes “the technician doesn’t see carefully.” Some health workers indicated that current training for technicians was too short and thus untrustworthy; training typically lasted only a few months rather than the "many years" considered necessary by these participants. Hence, a health worker's own judgment was commonly held to be better than the diagnostic service available.

With regard to RDTs, doubts expressed over the reliability of a negative test result were commonly framed around the test as a new technology. The newness of RDTs, particularly in comparison with microscopy, was deemed by some health workers as indicative of potential unreliability. According to a doctor from the northern province, “Time will prove whether ... RDTs will be reliable or not.” This emerged more clearly 
among those health workers who had been using microscopy for a longer time. However, these doubts could also reflect interpretations of the ongoing trial in which health workers were participating. Although trial participants received standard training using current guidelines (World Health Organization, 2006), which included a description of the accuracy of the tests, a few health workers referred explicitly to RDTs as the subject of the trial when describing their doubts about test results. A nurse from the northern province suggested that because "it is in study . . . it isn’t one hundred percent correct to trust RDT.” Here, it appears that the trial might have been interpreted by some as a trial of effectiveness of the technology, thus influencing the perceived reliability of the test results.

Contextualized treatment. As well as the logic of a clinical diagnosis of malaria following a negative test, and doubts around the reliability of tests, several contextual factors were identified as supporting health workers' prescriptions of antimalarial drugs. These included weighing risks, logistics, and social issues. Many health workers perceived malaria to be highly prevalent in the local area, and therefore important to treat with antimalarial drugs even following a negative test. The danger of overuse of medicines articulated by some was countered by others who suggested antimalarial drugs were relatively harmless. It was conveyed by several participants that in some cases, because of traveling distance, patients should be treated presumptively in case they could not return quickly, as stated by an assistant doctor from the eastern province:

Our countryside is very big, so . . . if we have made a wrong diagnosis, and this man has walked for three or four hours and has come to the clinic.... If he does not have malaria we should give him antimalarials because this man would not die because of antimalarials, but if this patient has malaria, and then again comes back to us, so maybe we lose this patient.

In addition, the relationship between the health worker and the patient appeared to influence decisions around the prescription of antimalarial drugs. Although most participants stated explicitly that patients’ expectations or demands did not influence their practice, in more narrative accounts of experiences providing care the picture was more nuanced. For some health workers, anxiety about being respected and trusted by the patient was identified as an influencing factor in fulfilling patients' expectations or demands for antimalarial drugs. One doctor from the eastern province identified "soothing the patient" by meeting demands for medicines as important for ensuring "they have trust in the doctor." 
The concept of “serving the people” was emphasized by many participants as a primary driver for being a health worker. It was commonly conceptualized in terms of the health worker's responsibility to serve local communities and Allah [God], as well as individual patients. A doctor from the eastern province stated, So it's like this, that Allah has made human the best of all creatures, and as [medicine] is a service for humans, our aim and purpose in medicine was that we should become the source of service for the best creature of Allah, so that is why we turned to this side.

Associated with being able to serve the people through medicine were notions of prestige of the position of doctor within a community, whereby doctors, according to one from the northern province, "will be respected" by the people. Health workers articulated, however, that these values were often undermined by the lack of resources within the health system; for example, shortages of drugs and equipment, and ongoing political and armed conflict that affected the ability to travel and the availability of clinical staff.

\section{Negotiating Diagnosis Priorities}

Finally, it emerged from participants’ accounts that their perceptions of and responses to malaria testing were located within broader ideas of the position of the disease, and guidance on its diagnosis and treatment in relation to other priorities in their practice. Alongside many health workers' (correct) perceptions that the risk of malaria had reduced in recent years, participants recognized a range of other diseases as being common, and of concern to both patients and health workers. Frequently, when asked to describe a "typical patient” at their facility, participants drew on a list of various different diseases or conditions, not always including malaria. Typhoid, pneumonia, mental health and maternal health issues, along with a host of other conditions, featured in many health workers' conceptualizations of their patient population and usual clinical activities, as described by a doctor from the eastern province: "Many patients visit us. . . . We see . . . common cold[s], ... malaria, tuberculosis, mental health, injuries, hypertension, heart diseases, gastric . . . patients.”

The multiplicity of conditions that health workers encountered in their practices meant that fever and malaria competed with a wide variety of other possible diagnoses within ordinary clinical practice. This added to the broader context of uncertainty within which health workers operated, and was compounded by the lack of diagnostic tools for other conditions. Many participants reflected on and lamented the absence of tools for diagnosing conditions other than malaria. For example, a community health supervisor from the northern province remarked, “This laboratory is only for malaria now, not for typhoid, hepatitis, or others. If a general laboratory [were to] exist for this clinic, it would have a good result.” 
Hence, reliance on clinical judgment remained a fundamental part of health workers' practice. This was reflected in participants’ perceptions of the reliability of their clinical skills. Clinical judgment had primacy above diagnostic tests in the process of excluding other diseases to reach a diagnosis of clinical malaria. A doctor from the northern province exemplified this:

I: When do you think that your clinical diagnosis is more correct than the test result?

P: In cases when the patient has typical signs and symptoms of malaria and I don't find any other reason for a patient's fever. It means when I don’t see any other cause for a patient's fever and the patient has all the typical signs and symptoms of malaria, and the region is under risk of malaria. In this case ... my clinical diagnosis will be correct.

As reflected in the above quotation, many health workers referred to the "signs and symptoms” of malaria as a generalized, compound category and rarely discussed specific components of this group. When asked explicitly about symptoms, however, a small number of participants suggested that they would ask a patient about the pattern of fever they had experienced when trying to come to a diagnosis. This might have reflected the tertian cycle of fever and chills commonly associated with vivax infection. A nurse from the eastern province described investigating fever: "We will ask the patient about his fever, since when he has been suffering from fever. In malaria diseases fever is intermittent and in other diseases fever may be continuous.”

Levels of awareness and knowledge of guidance on managing malaria varied among the participants. Most health workers stated that they followed "national policy" for malaria in their clinic, but description of the content of malaria control policy was often vague, with little awareness of specific guidelines. This was demonstrated by a nurse from the northern province, who said, "The national policy is ... to decrease malaria in our country by RDT, microscopy, bed net, or any other possible way.” In addition, health workers identified various different sources of guidance, including the World Health Organization, the Ministry of Public Health. and nongovernmental organizations. Some participants referred to guidelines when describing what they would prescribe to a patient with malaria, particularly those patients considered to be special; for example, young children or pregnant women. Thus, it appeared that guidelines were largely interpreted as tools to inform the prescription of medicines following a malaria diagnosis, rather than to inform the process of diagnosis itself.

\section{Discussion}

Afghan health workers in this study appeared to practice concurrently both diagnostic-based and clinical malaria diagnosis. This occurred in a context in which, as indicated by recent observational data, overdiagnosis and 
overtreatment of malaria were widespread. This was particularly the case where no diagnostic technology was available, but also in locations where health workers had access to microscopy and/or RDTs (Leslie et al., 2012).

The introduction into a health system of RDTs for malaria diagnosis can be seen to follow the "technological imperative” in which problems are viewed as manageable through technological solutions (Barger-Lux \& Heaney, 1986). The discourse of those developing policy and guidelines is one that encourages the uptake of diagnostic tools for malaria at a global level. Thus, it reflects the discourse of the "biotechnical embrace" whereby quality of health care is equated with use of the newest technologies, which are interpreted as objects of hope in biomedicine (DelVecchio Good, 2001, 2010). Although this might be the case for those setting policy guidelines and delivering programs with the central recommendation of treatment for parasitologically confirmed malaria (World Health Organization, 2010a), the story for those expected to integrate diagnostic tests into their practice was more complex.

In the interviews, Afghan health workers conveyed two different conceptualizations of the value of tests. When asked directly about tests, they described and were cautiously enthusiastic about potential benefits of using RDTs and microscopy in terms of the technologies' abilities to confirm their clinical judgment. This was perceived to facilitate more effective treatment of the patient, to bring satisfaction to patients, and to offer increased access to better care. These benefits were important in a context in which resources were severely limited and the poorest and most vulnerable faced the most difficulties in accessing care (Trani et al., 2010).

In participants' narrative accounts of their experiences of engagement with these tools, however, it was suggested that the "imperative" of diagnostic technologies was not central to their practice. Rather, clinical judgment remained the imperative above enthusiasm for the potential of the tests. The construct of the confirmed diagnosis illustrated this point, with the value of the test not independent of, but linked to its ability to support the health worker's own clinical judgment. Furthermore, a negative test result—interpreted as an absence of diagnosis—appeared to create a space in which clinical diagnosis of malaria and subsequent treatment with antimalarial drugs was legitimate.

These findings demonstrated a difference in the engagement with the technological imperative between policy or program makers and the health workers in our study. This could reflect country-specific factors and/or more general differences between those who make and those who enact health care policies. In Afghanistan, the retention of clinical judgment as imperative above technology might reflect the backdrop of medical training underpinning health workers’ practice—-historically centered on clinical judgment—as well a lack of tests and 
clinical algorithms for alternative, nonmalarial febrile illnesses. Guidelines on using diagnostic technologies do not offer clear advice on how to negotiate the lack of diagnosis offered by a negative test result. This is particularly concerning for contexts in which malaria represents a small minority of the burden of disease in the face of multiple other possible diagnoses (Howard et al., 2010; Morikawa et al., 2011).

The contradiction of the discourse of the technological imperative is salient beyond the context of this study. The technological imperative is undermined when developments in biomedical science, including new technologies, do not eliminate health workers' uncertainty in their practice, but produce more questions. As a result, health workers resort to that which is reassuring to them (Fox, 1980): typically clinical judgment. For example, a preference for clinical judgment over new diagnostic technologies, reflecting long-standing local guidelines for presumptive malaria treatment, was found in Cameroon (Chandler et al., 2012).

The primacy of the health workers' clinical judgment in Afghanistan was reinforced by doubts about the reliability of microscopy and RDTs. These were (correctly) perceived as vulnerable to the actions of parasites, patients, and laboratories, and also to environmental conditions. This coincides with the findings of other studies exploring health workers’ nonadherence to test results in Africa (Kyabayinze et al., 2010; Perkins \& Bell, 2008). Lack of faith in the tests can also be seen as a response to a broader context of uncertainty of resources and the quality of service provision within the health system as a whole. In other diagnostics literature, particularly HIV testing, attempts to manage uncertainty around testing have been interpreted as ways to deal with greater "existential uncertainties" around disease and its position in the local social, cultural, and political environments (Kielmann et al., 2005).

In Afghanistan, provision of public health care is underresourced and occurs in highly challenging geographical and political circumstances. This, plus a total dependence on external aid for health care resourcing, has led to uncertainty of the sustainability of care (Sabri, Siddiqi, Ahmed, Kakar, \& Perrot, 2007). In this environment of conflict, political instability, and hardship (Eggerman \& Panter-Brick, 2010), the notion of existential uncertainty faced by health workers has strong resonance. Thus, it is important to interpret health workers' attempts to moderate the role of diagnostic technology in clinical practice as not only an expression of doubt over the reliability of the technology itself, but also the delivery of health care as a whole.

The cultural position of the health worker, in relation to patients and the surrounding social context, influenced the decision-making process for malaria diagnosis and treatment. Participants' expressions of their responsibility as health workers to serve the people underpinned the negotiation of the clinical encounter and testing for malaria. Service is held as an important aspiration for many people in Afghanistan, and the concept 
of social responsibility_-being "mashour"—is characterized by working hard to serve the community and the people in general (Eggerman \& Panter-Brick, 2010). Positions of leadership and prestige in Afghan society are constructed around perceived honesty, fairness, and trustworthiness, but also the ability to provide resources to the local community (Wakefield \& Bauer, 2005).

Reflecting this, a health worker faces a complex set of decisions and responsibilities when negotiating the diagnosis of malaria and prescription of medicines. There exists the importance of satisfying the individual patient clinically, but also socially in terms of providing him or her with resources including a test, diagnosis, and treatment. Against this, however, runs the responsibility to serve the wider community. In this study, health workers acknowledged their role in managing limited resources to serve the longer-term interests of the community at large.

\section{Limitations}

The interpretations presented in this article were the result of analysis of transcripts that were translated, so specific cultural meaning and nuances might have been lost or misinterpreted. The transcripts were coded by a separate team of researchers to those who carried out the fieldwork, impacting interpretive ability. Care was taken to limit interpretation problems by adopting a meaning-based method of translation and using a process of cross-checking with bilingual Pashto, Dari, and English speakers. Interpretations were cross checked with those based in the field throughout the processes of data collection, analysis, and writing, and revisions were made to data collection tools, coding, and the final analyses accordingly. The generalizability of these findings is limited to an extent to the Afghan setting, although the commonalities with study findings from elsewhere suggest many of the themes might be relevant to other low-resource settings.

\section{Recommendations}

Expanded use of microscopy and RDTs stand to improve patients’ access to parasitological diagnosis of malaria and reduce unnecessary prescription of antimalarial drugs. However, the strategies adopted to roll out these interventions and the accompanying guidance to health workers must take into consideration the practice of malaria diagnosis in relation to other clinical priorities in specific settings. A blanket approach of wide-scale recommendation of diagnostic testing, embedded in an assumed shared ideal of the technological imperative, has clear limitations in the context of Afghanistan. The social and historical importance of the clinical imperative in this setting should be acknowledged. Guidelines should be specifically developed for this setting that avoid placing diagnostic technology at the center of the care process, and that indicate how best microscopy and RDTs can be used to support health workers’ clinical judgment. 
Furthermore, for the Afghan context, as for Africa, guidelines must acknowledge that the use and interpretation of diagnostic tests do not occur within a clinical vacuum but are always located within a broader, often shifting context of competing material and social resources. The decision to treat is based not only on the test result, but is also bound to the responsibilities of the health worker toward the wider community, as well as the individual patient. Greater efforts should be made to understand how best to encourage health workers to negotiate the lack of diagnosis presented by a negative test result, and to support strategies that facilitate the diagnosis of other febrile illnesses which are often of higher priority for the health worker and in public health terms. In this study, the influence of the wider social, political, and material environment on the provision of health care was evident, and should be used as an opportunity to explore more nuanced and context-specific approaches to reduce overdiagnosis of malaria and improve treatment of nonmalarial fever.

\section{Acknowledgments}

We gratefully acknowledge the valuable contribution of Miriam Kayendeke to the coding of data in this study.

\section{Declaration of Conflicting Interests}

The authors declared no potential conflicts of interest with respect to the research, authorship, and/or publication of this article.

\section{Funding}

The authors disclosed receipt of the following financial support for the research, authorship, and/or publication of this article: The study was funded by the ACT Consortium through a grant to the London School of Hygiene \& Tropical Medicine from the Bill \& Melinda Gates Foundation. 


\section{References}

Adimi, F., Soebiyanto, R.P., Safi, N., \& Kiang, R. (2010). Towards malaria risk prediction in Afghanistan using remote sensing. Malaria Journal, 9, 125. doi:10.1186/1475-2875-9-125

Afghanistan Independent Human Rights Commission. (2011). Fifth report: Situation of economic and social rights in Afghanistan. Kabul, Afghanistan: Author. Retrieved from http://www.aihrc.org.af/media/files/Reports/SECR/Report\%20on\%20ESCR_Final_English_12_2011.p $\underline{\mathrm{df}}$

Ansah, E.K., Narh-Bana, S., Epokor, M., Akanpigbiam, S., Quartey, A. A., Gyapong, J., \& Whitty, C.J. (2010). Rapid testing for malaria in settings where microscopy is available and peripheral clinics where only presumptive treatment is available: A randomised controlled trial in Ghana. British Medical Journal, 340, c390. doi:10.1136/bmj.c930

Auerbach, C., \& Silverstein, L. (2003). Qualitative data: An introduction to coding and analysis. New York: New York University Press.

Barger-Lux, M. J., \& Heaney, R. P. (1986). For better and worse: The technological imperative in health care. Social Science \& Medicine, 22(12), 1313-1320. doi:10.1016/0277-9536(86)90094-8

Bisoffi, Z., Gobbi, F., Angheben, A., \& Van den Ende, J. (2009). The role of rapid diagnostic tests in managing malaria. PLoS Medicine, 6(4), e1000063. doi:10.1371/journal.pmed.1000063

Brooker, S., Leslie, T., Kolaczinski, K., Mohsen, E., Mehboob, N., Saleheen, S., . . Kolaczinski, J. (2006). Spatial epidemiology of Plasmodium vivax, Afghanistan. Emerging Infectious Diseases, 12(10), 16001602. doi:10.3201/eid1210.060051

Chandler, C., Jones, C., Boniface, G., Juma, K., Reyburn, H., \& Whitty, C. J. (2008). Guidelines and mindlines: Why do clinical staff over-diagnose malaria in Tanzania? A qualitative study. Malaria Journal, 7, 53. doi:10.1186/1475-2875-7-53

Chandler, C., Mangham, L., Njei, A. N., Achonduh, O., Mbacham, W. F., \& Wiseman, V. (2012). 'As a clinician, you are not managing lab results, you are managing the patient': How the enactment of malaria at health facilities in Cameroon compares with new WHO guidelines for the use of malaria tests. Social Science \& Medicine, 74(10), 1528-1535. doi:10.1016/j.socscimed.2012.01.025

Chandler, C., Whitty, C. J., \& Ansah, E. K. (2010). How can malaria rapid diagnostic tests achieve their potential? A qualitative study of a trial at health facilities in Ghana. Malaria Journal, 9, $\underline{95}$. doi:10.1186/1475-2875-9-95 
Chandler, C., Wood, M., Reynolds, J., Mikhail, A., Ahmad, T., Karimullah, K., .. . Leslie, T. How does patient expectation influence prescription practice in Afghanistan and what influences patient satisfaction? Manuscript in preparation

Cohen, J., Dupas, P., \& Schaner, S. (2011). Price subsidies, diagnostic tests, and targeting of malaria treatment: Evidence from a randomized controlled trial. Boston: Harvard School of Public Health. Retrieved from http://harvardschoolofpublichealth.org/faculty/jessicacohen/files/cohendupasschaner_act_16jun2011.pdf

DelVecchio Good, M.-J. (2001). The biotechnical embrace. Culture, Medicine and Psychiatry, 25(4), 395-410. doi:10.1023/a:1013097002487

DelVecchio Good, M.-J. (2010). The medical imaginary and the biotechnical embrace: Subjective experiences of clinical scientists and patients. In B. J. Good, M. Fischer, S. Willen, \& M.-J. DelVecchio Good (Eds.), A reader in medical anthropology: Theoretical trajectories, emergent realities (pp. 272-283). Chichester, UK: Wiley-Blackwell.

Eggerman, M., \& Panter-Brick, C. (2010). Suffering, hope, and entrapment: Resilience and cultural values in Afghanistan. Social Science \& Medicine, 71(1), 71-83. doi:10.1016/j.socscimed.2010.03.023

Fox, R. C. (1980). The evolution of medical uncertainty. Milbank Memorial Fund Quarterly: Health and Society, 58(1), 1-49. Retrieved from http://www.jstor.org/

Gardner, J., Dew, K., Stubbe, M., Dowell, T., \& Macdonald, L. (2011). Patchwork diagnoses: The production of coherence, uncertainty, and manageable bodies. Social Science \& Medicine, 73(6), 843-850. doi:10.1016/j.socscimed.2010.12.010

Guerra, C. A., Howes, R. E., Patil, A. P., Gething, P. W., Van Boeckel, T. P., Temperley, W. H., . . Hay, S. I. (2010). The international limits and population at risk of Plasmodium vivax transmission in 2009. PLoS Neglected Tropical Diseases, 4(8), e774. doi:10.1371/journal.pntd.0000774

Harvey, S., Jennings, L., Chinyama, M., Masaninga, F., Mulholland, K., \& Bell, D. (2008). Improving community health worker use of malaria rapid diagnostic tests in Zambia: Package instructions, job aid and job aid-plus-training. Malaria Journal, 7, 160. doi:10.1186/1475-2875-7-160

Howard, N., Chandramohan, D., Freeman, T., Ahmed, S., Mohammed, R., Sayed, E., \& Rowland, M. (2003). Socio-economic factors associated with the purchasing of insecticide-treated nets in Afghanistan and their implications for social marketing. Tropical Medicine \& International Health, 8(12), 1043-1050. doi:10.1046/j.1365-3156.2003.01163.x 
Howard, N., Shafi, A., Jones, C., \& Rowland, M. (2010). Malaria control under the Taliban regime: Insecticidetreated net purchasing, coverage, and usage among men and women in eastern Afghanistan. Malaria Journal, 9, 7. doi:10.1186/1475-2875-9-7

Kielmann, K., Deshmukh, D., Deshpande, S., Datye, V., Porter, J., \& Rangan, S. (2005). Managing uncertainty around HIV/AIDS in an urban setting: Private medical providers and their patients in Pune, India. Social Science \& Medicine, 61(7), 1540-1550. doi:10.1016/j.socscimed.2005.02.008

Kyabayinze, D., Asiimwe, C., Nakanjako, D., Nabakooza, J., Counihan, H., \& Tibenderana, J. (2010). Use of RDTs to improve malaria diagnosis and fever case management at primary health care facilities in Uganda. Malaria Journal, 9, 200. doi:10.1186/1475-2875-9-200

Leslie, T., Mayan, M. I., Hasan, M. A., Safi, M. H., Klinkenberg, E., Whitty, C. J., \& Rowland, M. (2007). Sulfadoxine-pyrimethamine, chlorproguanil-dapsone, or chloroquine for the treatment of Plasmodium vivax malaria in Afghanistan and Pakistan: A randomized controlled trial. Journal of the American Medical Association, 297(20), 2201-2209. doi:10.1001/jama.297.20.2201

Leslie, T., Mikhail, A., Mayan, I., Anwar, M., Bakhtash, S., Nader, M., Chandler, C., Whitty, C.J.M., Rowland, M. (2012). Overdiagnosis and mistreatment of malaria among febrile patients at primary healthcare level in Afghanistan: observational study. British Medical Journal, 345:e4389. doi:10.1136/bmj.e4389

Lock, M., \& Nguyen, V. (2010). An anthropology of biomedicine. Chichester, UK: Wiley-Blackwell.

Lubell, Y., Reyburn, H., Mbakilwa, H., Mwangi, R., Chonya, S., Whitty, C. J., \& Mills, A. (2008). The impact of response to the results of diagnostic tests for malaria: Cost-benefit analysis. British Medical Journal, 336(7637), 202-205. doi:10.1136/bmj.39395.696065.47

Ministry of Public Health. (2007). Draft national malaria strategic plan 2008-2013. Kabul, Afghanistan:

Author. Retrieved from

http://moph.gov.af/Content/Media/Documents/DraftNationalMalariaStrategicPlan2008201329920122055633553325325.pdf

Mol, A. (2002). The body multiple: Ontology in medical practice. Durham, NC: Duke University Press.

Morikawa, M. J., Schneider, S., Becker, S., \& Lipovac, S. (2011). Primary care in post-conflict rural northern Afghanistan. Public Health, 125(1), 55-59. doi:10.1016/j.puhe.2010.08.021

Nichter, M., \& Vuckovic, N. (1994). Agenda for an anthropology of pharmaceutical practice. Social Science \& Medicine, 39(11), 1509-1525. doi:10.1016/0277-9536(94)90003-5 
Perkins, M., \& Bell, D. (2008). Working without a blindfold: The critical role of diagnostics in malaria control. Malaria Journal, 7(Suppl. 1), S5. doi:10.1186/1475-2875-7-S1-S5

Reyburn, H., Mbakilwa, H., Mwangi, R., Mwerinde, O., Olomi, R., Drakeley, C., \& Whitty, C. J. (2007). Rapid diagnostic tests compared with malaria microscopy for guiding outpatient treatment of febrile illness in Tanzania: Randomised trial. British Medical Journal, 334, 403. doi:10.1136/bmj.39073.496829.AE

Reynolds Whyte, S., Van der Geest, S., \& Hardon, A. (2002). Social lives of medicines. Cambridge: Cambridge University Press.

Rowland, M., Mohammed, N., Rehman, H., Hewitt, S., Mendis, C., Ahmad, M., . . Wirtz, R. (2002). Mosquito vectors and malaria transmission in eastern Afghanistan. Transactions of the Royal Society of Tropical Medicine \& Hygiene, 96, 620-626. doi:10.1016/S0035-9203(02)90331-7

Sabri, B., Siddiqi, S., Ahmed, A. M., Kakar, F. K., \& Perrot, J. (2007). Towards sustainable delivery of health services in Afghanistan: Options for the future. Bulletin of the World Health Organization, 85(9), 712718. doi:10.2471/BLT.06.036939

Strauss, A., \& Corbin, J. (1998). Basics of qualitative research techniques and procedures for developing grounded theory (2nd ed.). London: Sage.

Trani, J. F., Bakhshi, P., Noor, A. A., Lopez, D., \& Mashkoor, A. (2010). Poverty, vulnerability, and provision of healthcare in Afghanistan. Social Science \& Medicine, 70(11), 1745-1755. doi:10.1016/j.socscimed.2010.02.007

Wakefield, S., \& Bauer, B. (2005). A place at the table: Afghan women, men and decision-making authority. Kabul: Afghanistan Research and Evaluation Unit. Retrieved from http://www.areu.org.af/EditionDetails.aspx?EditionId=29\&ContentId=7\&ParentId=7

Whitty, C. J., Chandler, C., Ansah, E. K., Leslie, T., \& Staedke, S. (2008). Deployment of ACT antimalarials for treatment of malaria: Challenges and opportunities. Malaria Journal, 7(Suppl. 1), S7 doi:10.1186/1475-2875-7-s1-s7

World Health Organization. (2006). The use of rapid malaria diagnostic tests (2nd ed.). Geneva: Author. Retrieved from http://www2.wpro.who.int/sites/rdt/documents/pub_9290612045.htm

World Health Organization. (2010a). Guidelines for the treatment of malaria (2nd ed.). Geneva: Author. Retrieved from http://whqlibdoc.who.int/publications/2010/9789241547925_eng.pdf World Health Organization. (2010b). World malaria report 2010. Geneva: Author. Retrieved from http://www.who.int/entity/malaria/world_malaria_report_2010/worldmalariareport2010.pdf 
World Health Organization. (2010c). World malaria report country profile-Afghanistan. Geneva: Author.

Retrieved from http://www.who.int/malaria/publications/country-profiles/profile_afg_en.pdf

\section{Bios}

Joanna Reynolds, MSc, is a research fellow in social science in the Department of Global Health \&

Development, and a social scientist for the ACT Consortium at the London School of Hygiene \& Tropical Medicine in London, United Kingdom.

Molly Wood, MSc, is a social science researcher for the Health Protection and Research Organisation in Kabul, Afghanistan.

Amy Mikhail, MSc, is a research fellow for the ACT Consortium at the London School of Hygiene \& Tropical Medicine in London, United Kingdom, and the laboratory technical advisor to Health Protection and Research Organisation in Kabul, Afghanistan.

Tamanna Ahmad is a social science field officer for the Health Protection and Research Organisation in Kabul, Afghanistan.

Karimullah Karimullah, BSc, is a social science field officer for the Health Protection and Research Organisation in Kabul, Afghanistan.

Mohibullah Motahed, BSc, is a social science field officer for the Health Protection and Research Organisation in Kabul, Afghanistan.

Anwar Hazansai, MD, is the ACT Consortium project coordinator for HealthNet TPO in Kabul, Afghanistan.

Sayed Habib Baktash, MD, is the ACT Consortium project manager for MERLIN in Kabul, Afghanistan.

Nadia Anwari is a social science field officer for the Health Protection and Research Organisation in Kabul, Afghanistan.

James Kizito, BA, is a research assistant in social science for the Infectious Diseases Research Collaboration in Kampala, Uganda.

Ismail Mayan, MD, is the ACT Consortium project coordinator for the Health Protection and Research Organisation in Kabul, Afghanistan.

Mark Rowland, PhD, is head of the Department of Disease Control at the London School of Hygiene \& Tropical Medicine in London, United Kingdom.

Clare Chandler, MSc, PhD, is a lecturer in social science in the Department of Global Health \& Development, and lead social scientist for the ACT Consortium at the London School of Hygiene \& Tropical Medicine in London, United Kingdom. 
Toby Leslie, MSc, PhD, is a lecturer in the Department for Disease Control, the policy and programmes liaison coordinator for the ACT Consortium at the London School of Hygiene \& Tropical Medicine in London, United Kingdom, and technical Director of the Health Protection and Research Organisation in Kabul, Afghanistan.

Table 1. Characteristics of Health Workers Participating in Interviews

\begin{tabular}{|c|c|c|c|}
\hline \multirow[t]{2}{*}{ Participant Characteristics } & \multirow[t]{2}{*}{ Total Sample } & \multicolumn{2}{|c|}{ Participants by Province } \\
\hline & & Eastern & Northern \\
\hline Total number & 39 & 22 & 17 \\
\hline \multicolumn{4}{|l|}{ Gender } \\
\hline Male & 35 & 18 & 17 \\
\hline Female & 4 & 4 & 0 \\
\hline \multicolumn{4}{|l|}{ Health worker grade } \\
\hline Doctor & 23 & 15 & 8 \\
\hline Assistant doctor & 1 & 1 & 0 \\
\hline Nurse & 11 & 6 & 5 \\
\hline Medical assistant & 3 & 0 & 3 \\
\hline Community health supervisor & 1 & 0 & 1 \\
\hline \multicolumn{4}{|l|}{ Diagnostic methods available } \\
\hline RDTs $^{\mathrm{a}}$ / established microscopy & 22 & 22 & 0 \\
\hline RDTs / new microscopy & 11 & 0 & 11 \\
\hline RDTs / clinical judgment & 6 & 0 & 6 \\
\hline Mean years' of professional experience (range) & $12.1(2-33)$ & $12.0(2-26)$ & $12.1(2-33)$ \\
\hline
\end{tabular}

${ }^{a}$ Rapid diagnostic tests 